\title{
Comparison of Nodule Endophyte Composition, Diversity, and Gene Content Between Medicago truncatula Genotypes
}

\author{
Mannix Burns, Brendan Epstein, and Liana T. Burghardt ${ }^{\dagger}$ \\ Department of Plant and Microbial Biology, University of Minnesota, St. Paul, MN 55108
}

Accepted for publication 21 May 2021.

\section{ABSTRACT}

Leguminous plants form symbiotic relationships with rhizobia. These nitrogen-fixing bacteria live in specialized root organs called nodules. Although rhizobia form the most notable host relationship within root nodules, other bacterial endophytes also inhabit nodules and can influence host-rhizobia interactions as well as exert effects of their own. In this study, we elucidate the effect of intraspecific host genetic variation and between generational legacies on root nodule endophyte communities in Medicago truncatula. Although the diversity of endophytes in nodules was similar across hosts, both nodule endophyte composition and gene functional groups differed. In contrast, neither the presence nor identity of a host in the previous generation (either A17 or R108) had a significant effect on the nodule endophyte diversity or composition. However, whether or not a host was present significantly altered gene functional groups. We conclude that genetic variation within a legume host species can play both direct and indirect effects on establishment of nodule microbiomes. Further studies, including genome-wide association studies and functional assays, can open the door for engineering and optimizing nodule endophyte communities that promote growth or have other beneficial qualities.

Keywords: endophytes, genotype-dependent effects, host-soil feedbacks, Medicago-Ensifer, microbiome, mutualism, phytobiomes, rhizosphere and phyllosphere, root nodule microbiome, symbiosis
Endophytic bacteria and fungi are common inhabitants of plant tissues such as roots and leaves, and often live in the soil when not infecting a host. In the case of legumes, nitrogen-fixing bacterial species (collectively called rhizobia) form symbiotic relationships with the host in specialized root organs called nodules (Long 1989). Although rhizobia are the primary bacterial inhabitants, other bacteria also inhabit nodules and have measurable phenotypic effects. For instance, coinoculation of common bean (Phaseolus vulgaris) with endophytic strains of Bacillus, Pseudomonas, or Burkholderia reduces damping-off (de Vasconcelos Martins Ferreira et al. 2020) while coinoculation with Agrobacterium reduces nodulation (Mhamdi et al. 2005; Mrabet et al. 2006). Endophytic bacteria in chickpea (Cicer

Current address of L. T. Burghardt: Department of Plant Science, The Pennsylvania State University, University Park, PA 16802

\section{Corresponding author: L. T. Burghardt; liana.burghardt@gmail.com}

Funding: This work was supported by the National Science Foundation under grant numbers IOS-1724993 and IOS-1856744 and the United States Department of Agriculture-National Institute of Food and Agriculture, Hatch project 1025611.

*The $e$-Xtra logo stands for "electronic extra" and indicates that a supplementary file and supplementary materials are published online.

The author(s) declare no conflict of interest.

C 2021 The American Phytopathological Society arietinum L.) have been shown to increase plant growth and suppress root rot (Egamberdieva et al. 2017). Although a few examples have been studied, overall, little is known about the identity and phenotypic effects of nodule endophytes despite their potential value as biocontrol agents (Martínez-Hidalgo and Hirsch 2017).

The composition of nodule microbiome communities differs between plant species, suggesting that genetic differences can have substantial effects on which bacteria inhabit nodules (Xiao et al. 2017). There is reason to suspect, based on data from other plant tissues, that genetic variation within a species could also play a role in determining nodule endophyte composition. For example, tomato cultivars differ in leaf microbiomes (Morella et al. 2020), and host genotype plays a role in the composition of the endophyte communities in natural populations of some plant species such as Boechera stricta (Wagner et al. 2016) and Populus balsamifera (Bálint et al. 2013) and agricultural cultivars of Zea mays (Ikeda et al. 2013). Furthermore, reverse genetics experiments indicate that genes involved in symbiosis may play a role in determining the composition of the microbial community in the roots, rhizosphere, and nodules (Zgadzaj et al. 2016). Previous studies in the legumes Medicago truncatula, Glycine max (soybean), and Vigna unguiculata (cowpea) have shown mixed results: plant host genotype influences nodule endophyte composition (Brown et al. 2020; Sharaf et al. 2019) but not nodule endophyte diversity (Leite et al. 2016). To our knowledge, no studies have tested for intergenerational effects of prior hosts on nodule endophyte communities-although both inter- and intraspecific plant genetic 
variation can clearly influence soil microbiome composition and function (Cloutier et al. 2020; Fischer et al. 2014). Due to the scarcity of studies and the broad phylogenetic range of host species tested, it is difficult to formulate a priori predictions. Here, we examine nodule endophyte communities in two accessions of the model legume $M$. truncatula and test for the effect of exposure to a previous generation of each host genotype.

$M$. truncatula harbors genetic variation for the diversity and composition of nitrogen-fixing Ensifer strains, also referred to as Sinorhizobium, with which they form nodules (Burghardt et al. 2018, 2019; Heath and Grillo 2016; Heath and Stinchcombe 2013). Burghardt et al. (2019) used a "select and resequence" experiment-whole-genome sequencing of rhizobia in pools of nodules - to measure the relative frequency of 101 coinoculated strains in two Medicago genotypes. The rhizobial community in the model genotype A17 was less diverse than and compositionally divergent from the rhizobial community in the model genotype R108 (Burghardt et al. 2019). In the same experiment, they found evidence that selective legacies of prior host genotypes exert effects on the diversity and composition of rhizobia in nodules - although the magnitude of the effect was far weaker than the direct effect of host genotype. We were curious whether these diversity and compositional differences extend to nonrhizobial bacteria in the nodules.

Here, we use the data from Burghardt et al. (2019) to elucidate the effect of intraspecific host genetic variation and the betweengenerational legacies of that genetic variation on root nodule endophyte communities in M. truncatula hosts. Specifically, we ask two questions. (i) Are there significant differences in overall diversity (the number and abundance of different endophytes), composition (the specific types of endophytes present), and gene content of the endophyte communities between the two hosts. (ii) Does the presence or identity of a prior host influence the overall diversity, composition, and gene content of the endophyte community in subsequent generations?

\section{MATERIALS AND METHODS}

Experimental design. We assessed nodule endophyte communities in two M. truncatula genotypes (A17 and R108) growing in the greenhouse (Fig. 1). A full description of the experimental design can be found in Burghardt et al. (2019). Soil and water were sterilized via autoclave (120-min liquid cycle) while seeds were surface sterilized for 30 to $45 \mathrm{~s}$ in $10 \%$ bleach, then rinsed with sterilized water. To confirm sterility, both water and a dilute soil slurry were plated on rich media to check for microbial growth. Briefly, we filled 30 1-liter pots with sterilized low-nitrogen peat soil (SunGro LP5; Bucciarelli et al. 2006) and inoculated each with a mixture of 101 previously sequenced Ensifer meliloti strains (approximately $10^{9}$ cells/pot). Sixteen of these pots were in the greenhouse for 12 weeks with no plants while seven pots were planted with 10 to 12 seeds of A17 and seven with 10 to 12 seeds of R108. Six weeks after planting, the aboveground vegetation was cut off and the roots and nodules were allowed to decompose for 6 weeks. Next, 10 to 12 sterilized and pregerminated seeds of either A17 or R108 were planted in the soil. Eight of the pots that had not been planted previously received A17 seed, and the other eight received R108 seed. All pots that previously had host plants were planted with R108 seed so the effect of prior selection could be measured in a common genotype. R108 followed by A17 and A17 followed by A17 treatments were not investigated due to sequencing and space limitations. The plants were allowed to grow and form nodules for 6 weeks before they were harvested, at which point all of the nodules were removed. These nodules were pooled, surface sterilized for 30 to $45 \mathrm{~s}$ in $10 \%$ bleach, rinsed with sterilized water four times, and crushed using a sterile pestle. The bacterial community (Ensifer strains + other endophytes) from the crushed nodules was separated through slow centrifugation to pellet plant debris and bacteroids ( $400 \times g$ for $10 \mathrm{~min}$ ) and fast centrifugation of the supernatant from the previous step $(10,000 \times g$ for $8 \mathrm{~min}$ ) to pellet the bacteria. Full details can be found in Burghardt et al. (2019).

As previously described, DNA was extracted from the crushed nodules and sequenced, and aligned reads were mapped to E. meliloti USDA1106 and used to assess strain frequencies of E. meliloti in nodules (Burghardt et al. 2019). In this article, we used two methods-BLAST and MEGAN6 (described below) - to map the unaligned reads (i.e., sequencing bycatch) to nonrhizobial endophytic bacteria.

BLASTing reads. We used BLAST to search the NCBI database for sequences with high similarity to the non-Ensifer and nonMedicago nodule reads, then processed the results using custom $\mathrm{R}$ and shell scripts (see Supplementary Materials). We randomly sampled 500,000 reads (out of approximately 1 to 4 million total) from each pot replicate that did not align to either the E. meliloti or M. truncatula reference genomes and used blastn to search bacterial accessions in the NCBI nt database; the maximum number of target sequences was set to 50 , and the maximum e-value was set to $10^{-5}$. For each read, we retained the highest-scoring hit. We filtered the reads further by disposing of all reads for which the best hit was to Sinorhizobium (a synonym of Ensifer) or unclassified bacteria which, as expected, eliminated approximately 90 to $97 \%$ of reads. After filtering, between 2.0 and $9.5 \%$ of the initial 500,000 reads came back with known classified hits to endophytic bacteria other than Sinorhizobium. The unmatched reads could be due to the presence of fungi or other microorganisms as well as bacteria without sequences in the NCBI database. We chose the best hit for every read and counted the number of reads with best matches by species, genus, and family (genus names were converted to family using the "taxize" $\mathrm{R}$ package). One sample—soil followed by R108 (soil $\rightarrow \mathrm{R} 108$ replicate 12 )—was removed as an outlier due to containing several orders of magnitude higher proportion of a single bacterial genus, Burkholderia, indicating an apparent infection.

Data subsets. Before testing for significant differences in endophyte composition, diversity, and function between treatments, we created four datasets. The first two subsets, used for most of the analysis, included all results from the BLAST search. The soil $\rightarrow$ A17 and soil $\rightarrow \mathrm{R} 108$ subset included replicates used to assess for direct effects

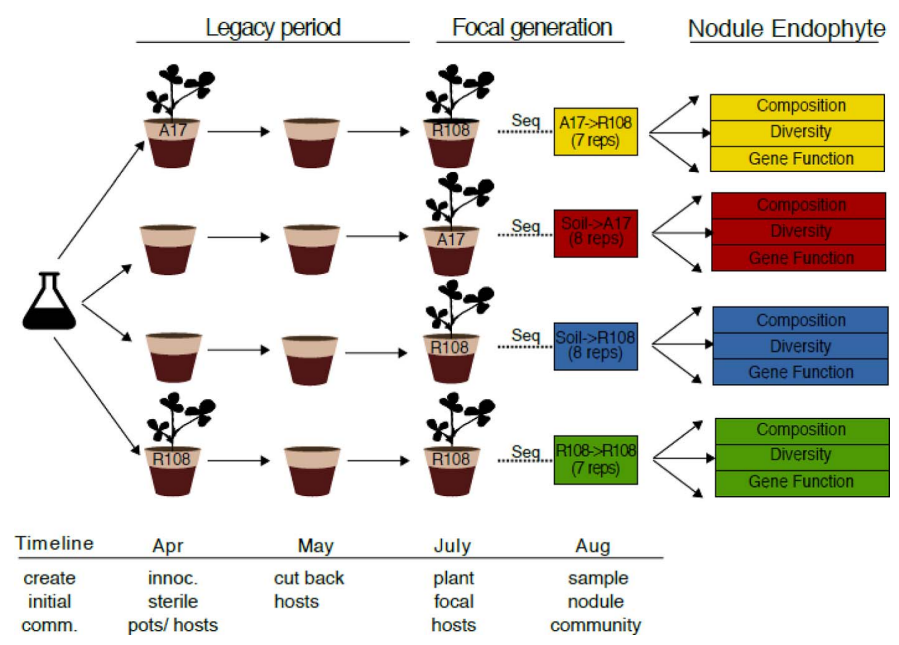

Fig. 1. Experimental design used to test for the direct effect of host genetic variation (soil $\rightarrow$ R108 versus soil $\rightarrow A 17$ ) and host genetic legacies $(A 17 \rightarrow R 108$ versus $R 108 \rightarrow R 108)$ on nodule endophyte communities. Figure modified from Burghardt et al. (2019). 
of host genotype. The second subset included all R108 replicates (soil $\rightarrow \mathrm{R} 108, \mathrm{~A} 17 \rightarrow \mathrm{R} 108$, and $\mathrm{R} 108 \rightarrow \mathrm{R} 108$ ) and was used to assess the indirect legacy effects. The soil $\rightarrow$ A17 and soil $\rightarrow$ R108 subset was further narrowed to focus on highly represented genera whose mean abundance was higher than $2 \%$ in at least one of the two host genotypes. This subset contained 15 bacterial families that represented 77 to $88 \%$ of the total community of each sample. This subset was used to investigate whether significant differences in composition between treatments were present in high abundance bacteria. An additional data subset of the top 15 bacterial families from all four treatments was used to visualize intra- and interhost endophyte composition.

Community composition. To visualize differences in endophyte composition across hosts, we used (i) nonmetric multidimensional scaling (metaMDS function in $\mathrm{R}$ with $k=2$ ), which measures changes in rank order of genera between different replicates, and (ii) BrayCurtis dissimilarity distance plots using the "vegdist" and "pco" functions (vegan package in R). We tested for significant differences among host genotypes using a permutational multivariate analysis of variance (PERMANOVA) on the Bray-Curtis distance matrix ("anova" and "adonis" functions in the vegan R package) as well as analyzing multivariate homogeneity of group dispersions ("betadisper" and "anova" in the vegan R package) to assess the microbiome consistency across replicates.

Community diversity metrics. To determine whether the diversity of endophyte communities differed between host genotypes, we quantified community diversity in each replicate using the Renyi diversity index ("renyi" function in the vegan package in R). We used the "anova" function in $\mathrm{R}$ to test for differences among host plant treatments at various scale parameters, including species richness $(\alpha=0)$, which only counts the number of species present, and Berger-Parker $(\alpha=\infty)$, which is maximized only when all species are present at equivalent abundances. We also tested two commonly used intermediate values where both species richness and evenness contribute to the index: exponent of Shannon's diversity $(\alpha=1)$ and Inverse Simpsons indices $(\alpha=2)$.

Automated taxonomic pipeline. To confirm the results gathered from the NCBI BLAST data, we ran our reads through an independent pipeline. We used DIAMOND (Buchfink et al. 2015), a highthroughput DNA read alignment program, to identify matching sequences in the NCBI nr protein database, and MEGAN6 (Huson et al. 2016), which maps the aligned reads to a taxonomic ID. As with the BLAST analysis, we removed any reads for which the hit was Sinorhizobium. After removing Sinorhizobium hits, 88 to $97 \%$ of the remaining reads had a match. We also used this pipeline to categorize genes into functional groups (gene ontology [GO] biological processes) for comparative analysis between genotypes. Using the "Interpro" function within MEGAN6, we mapped the aligned reads to Interpro (IPR) IDs which we then converted to GO biological processes using an R script (mapping file: ftp.ebi.ac.uk/pub/databases/ interpro/interpro2go). Only GO biological processes with at least 40 hits within a replicate were included. Both the taxa and GO term mapping files can be found on the MEGAN6 website (July 2019 mapping files were used in this analysis: https://software-ab.informatik.unituebingen.de/download/megan6/welcome.html). In addition, we tested for significant gene function enrichment differences between treatments using a PERMANOVA on a linear model for each functional gene category, then corrected $P$ values for multiple tests using a Bonferroni correction.

\section{RESULTS}

Effect of host genotype on endophyte community diversity. Our analysis of the root nodule microbial community identified
$>160$ bacterial families present in nodules. We found no evidence that endophyte diversity differed between the two host genotypes (Fig. 2A). This was the case both for metrics that emphasize the number of families such as species richness $\left(\mathrm{F}_{\mathrm{df}}=1=0.020, P=0.89\right)$ and Shannon diversity $\left(\mathrm{F}_{\mathrm{df}=1}=0.71, P=0.41\right)$, and those that emphasize community evenness such as Inverse Simpson's $\left(\mathrm{F}_{\mathrm{df}=1}=0.78, P=\right.$ $0.39)$ and Berger-Parker $\left(\mathrm{F}_{\mathrm{df}=1}=1.21, P=0.29\right)$. Thus, there are no significant differences in the diversity of the endophyte communities of the A17 and R108 M. truncatula genotypes. This conclusion held at the genus level as well (Supplementary Fig. S1). We also found no effect of host legacy treatment on endophyte diversity in nodules; species richness $(P=0.83)$, Shannon diversity $(P=0.43)$, Inverse Simpson's $(P=0.84)$, and Berger-Parker $(P=0.95)$ were all nonsignificant (Fig. 2B).

Compositional comparison of endophyte taxa between host genotypes. Although, at the endophyte family level, there are no significant differences in nodule community diversity, there are significant differences in composition between host genotypes (PERMANOVA, $\mathrm{F}_{\mathrm{df}=1}=3.1, R^{2}=0.19, P=0.003$ ) (Fig. 3). These differences can be visualized by both nonmetric multidimensional scaling (Supplementary Fig. S2) and principal coordinate analysis (Fig. 4A) of Bray Curtis dissimilarities. In addition to the compositional differences, there is weak evidence that the A17 replicates (Fig. 4A, red) are more compositionally consistent than R108 replicates (Fig. 4A, blue) (analysis of multivariate homogeneity of group dispersions, $\mathrm{F}_{\mathrm{df}=1}=3.8, P=0.073$ ). By contrast, at the genus level, there were significant differences between host genotypes (Supplementary Fig. S3) for both endophyte community composition $(P=0.001)$ and compositional consistency $(P=0.033)$.

Of $>160$ families (approximately $15 \%$ of known bacterial families), the 15 most abundant (mean frequency $>2 \%$ in at least one treatment) in each host genotype collectively comprised $>80 \%$ of the entire dataset. We tested for differences in community composition using just these 15 taxa to determine whether the differences between hosts were reflective of the behavior of abundant families. Consistent with the full dataset, there was a significant, direct effect of host genetic variation on community composition (PERMANOVA on the Bray-Curtis matrix, $\left.\mathrm{F}_{\mathrm{df}=1}=3.1, R^{2}=0.19, P=0.005\right)$. The bacterial families with the most substantial shifts in abundance between treatments included Xanthomonadaceae, Pseudomonadaceae, and Enterobacteriaceae, all of which were enriched in the soil $\rightarrow \mathrm{R} 108$ treatment. A larger sample size would be required to make claims about the significance of these shifts, some of which could be attributed to outliers. There was no evidence of a difference between A17 and $\mathrm{R} 108$ replicate dispersion $\left(\mathrm{F}_{\mathrm{df}=1}=3.9, P=0.69\right)$ (Fig. 3). At the genus level, there were significant differences between host genotypes for both endophyte community composition and compositional consistency among replicates in the abundant family dataset (Supplementary Fig. S4). Although the most common families detected by the MEGAN6 pipeline differed from those detected by the BLAST pipeline, both analyses revealed significant differences in community composition and no differences in replicate consistency between host genotypes (Supplementary Figs. S5 and S6), and the proportion of variance explained by host genotype was similar $\left(R_{\text {BLAST }}^{2}=0.19\right.$, $R_{\text {MEGAN }}^{2}=0.23$ ).

Because host genotype had a significant effect on endophyte community composition, we also tested whether the identity (A17 or R108) or presence of a previous host influenced nonrhizobial endophyte composition. Previously, we found that prior exposure to a host and the genetic identify of that host shifted Ensifer strain composition in nodules (Burghardt et al. 2019). Here, we found that neither of these legacies affected the nonrhizobial endophyte community at the family level (Fig. 4B) (PERMANOVA $\mathrm{F}_{\mathrm{df}}=1=1.27$, 
$\left.R^{2}=0.12, P=0.23\right)$ or the genus level (Supplementary Fig. S7) $\left(\mathrm{F}_{\mathrm{df}=1}=1.31, R^{2}=0.123, P=0.207\right)$.

Gene function comparison between host endophyte communities. Although compositional differences between host endophyte communities were sometimes evident at the taxonomic level, we also wanted to investigate whether there were differences between gene functional categories present in the endophyte communities. Using the relative abundance of GO biological process terms identified with MEGAN6, we found evidence of significant differences in gene functional groups between the soil $\rightarrow \mathrm{A} 17$ and soil $\rightarrow \mathrm{R} 108$ endophyte communities (PERMANOVA, $\mathrm{F}_{\mathrm{df}=1}=8.48, R^{2}=0.39, P=$ 0.001) (Fig. 5A). Thus, in addition to differing taxa composition, host genotypic differences have a direct effect on the abundance of functional gene categories in nodules. Interestingly, in contrast to the community composition analysis, host presence or absence in the previous generation had a significant effect on the abundance of functional gene groups (Fig. 5B) soil $\rightarrow$ R108 versus A17 $\rightarrow$ R108 combined with $\mathrm{R} 108 \rightarrow \mathrm{R} 108$ (PERMANOVA, $\mathrm{F}_{\mathrm{df}=1}=14.87, R^{2}=0.44$, $P=0.001)$. However, the identity of the prior host genotype had no effect $\left(\mathrm{A} 17 \rightarrow \mathrm{R} 108\right.$ versus $\mathrm{R} 108 \rightarrow \mathrm{R} 108, \mathrm{~F}_{\mathrm{df}=1}=1.71, R^{2}=0.12$, $P=0.068$ ).

In addition to testing for significant differences between the gene functional groups in the two host endophyte communities, we also investigated which of these functional groups were most significantly enriched within a treatment using PERMANOVA on a linear model for each functional gene category (Table 1). Enriched functions that differ across host genotypes include core energy metabolism; catabolism of aromatic compounds, carbohydrates, and pentose sugars; and sodium transport. Gene functional enrichment imposed by soil versus R108 legacies included DNA repair mechanisms, branched chain amino acid and sulfate transport, and fatty acid and formate biosynthesis. These functional differences between treatments likely depend on a variety of factors, including plant-microbe interactions, microbe-microbe interactions, and nutrient availability in the soil.
Because this experiment started from sterile media in a greenhouse rather than field soil, specific functional differences are difficult to contextualize.

\section{DISCUSSION}

We found negligible differences in the diversity of the root nodule endophyte community between two genotypes of M. truncatula, even though those two genotypes differ in rhizobial strain diversity (Burghardt et al. 2019). However, the two host genotypes differed significantly in the composition of their root nodule microbiome and in abundance of gene functional groups. By contrast, neither the presence of a prior host nor the genotypic identity of the prior host effected endophyte diversity, composition, or compositional variation among replicates. However, the presence of a host, regardless of genotype, influenced gene functional abundance. Our results suggest that the direct effect of host genetic differences is a stronger driver of nonrhizobial nodule endophyte communities than enrichment legacies from prior plants.

Comparisons of nodule endophyte communities in different legume species such as soybean and alfalfa have shown that host species does influence root nodule microbiomes (Xiao et al. 2017). However, not all tissues and plant species exhibit intraspecific microbiome variation. For example, $B$. stricta genotypes have significantly different microbiome communities in the leaves but not the roots (Wagner et al. 2016). Using sequencing bycatch from Burghardt et al. (2019), we extended findings from previous studies to show that genetic variation within a legume host species (M. truncatula) can also determine root nodule endophyte communities. The reuse of previously generated sequencing bycatch to ask new questions, as done here, has some precedent (Garcia et al. 2018) but is not common. However, going forward, we believe that this method will become increasingly commonplace as the amount of community level, whole-genome sequence data increases.
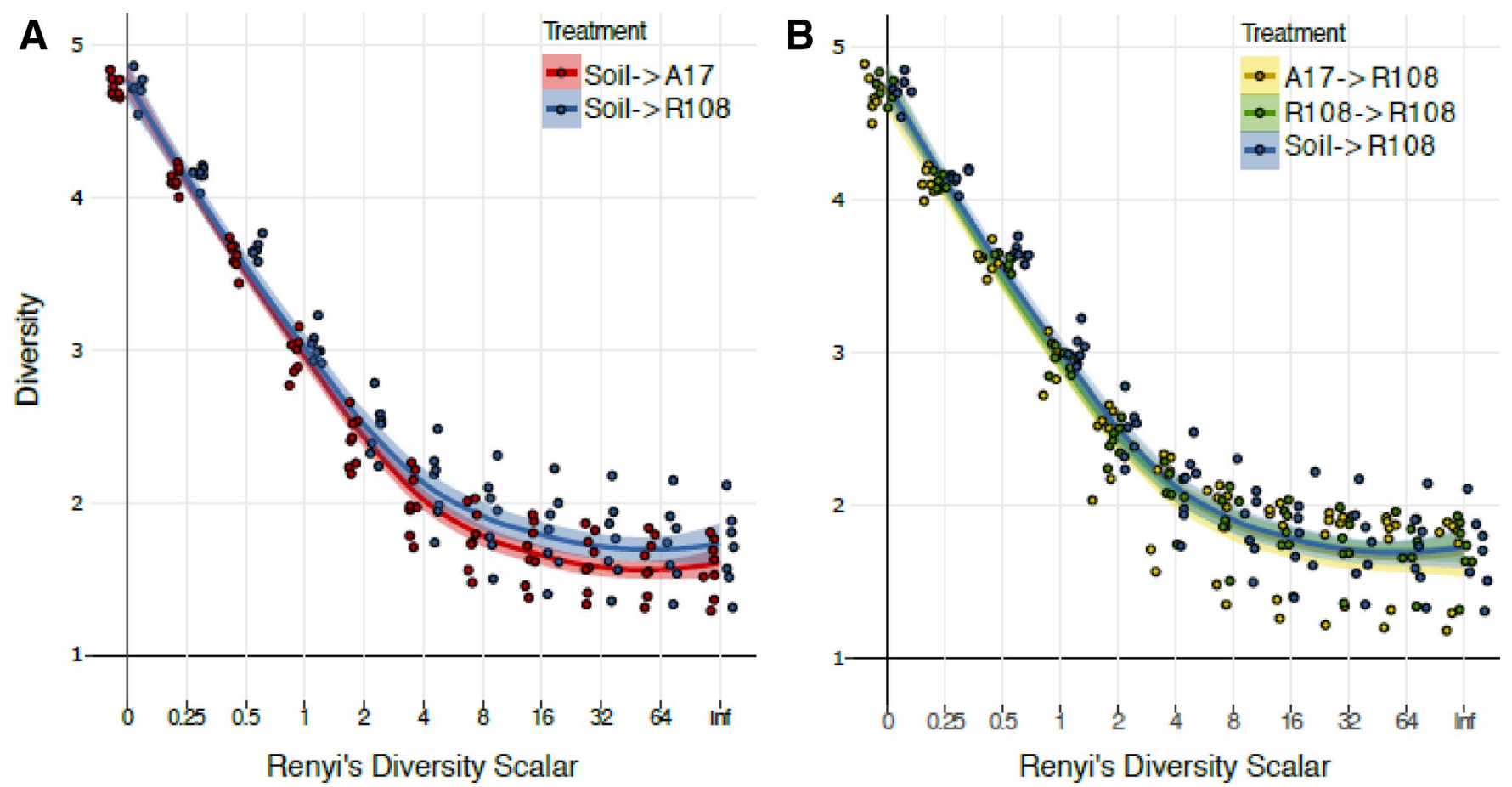

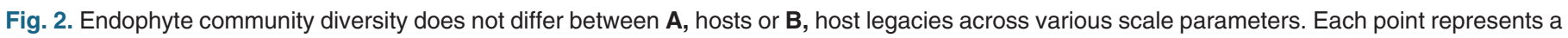
pot replicate, and we used a best-fit line to summarize trends across scale $\alpha$ parameters. Shaded areas represent a $95 \%$ confidence interval. Diversity metrics were calculated on the full family dataset from the blastn analysis. 
Within the scope of our experiment, we observed that a few bacterial families drove the compositional differences between the A17 and R108 hosts. Of note, species of Pseudomonadaceae appeared in higher frequency in all three R108 host treatments than in A17. Thus, the R108 genotype may be more susceptible to Pseudomonadaceae colonization than A17. Different Pseudomonadaceae species have different niches and can be both mutualists and pathogens
(Melnyk et al. 2019). Several species have growth-promoting properties as well as provide protection from certain parasitic fungi in plants (Haas and Défago 2005) and Caenorhabditis elegans (Zhang et al. 2017). Species-level data indicated that Pseudomonas aeruginosa, P. fluorescens, Pseudomonas sp. 31-12, and P. putida were the most prevalent species in our samples, with each being present across treatments. P. fluorescens (Hol et al. 2013), P. putida (Costa-

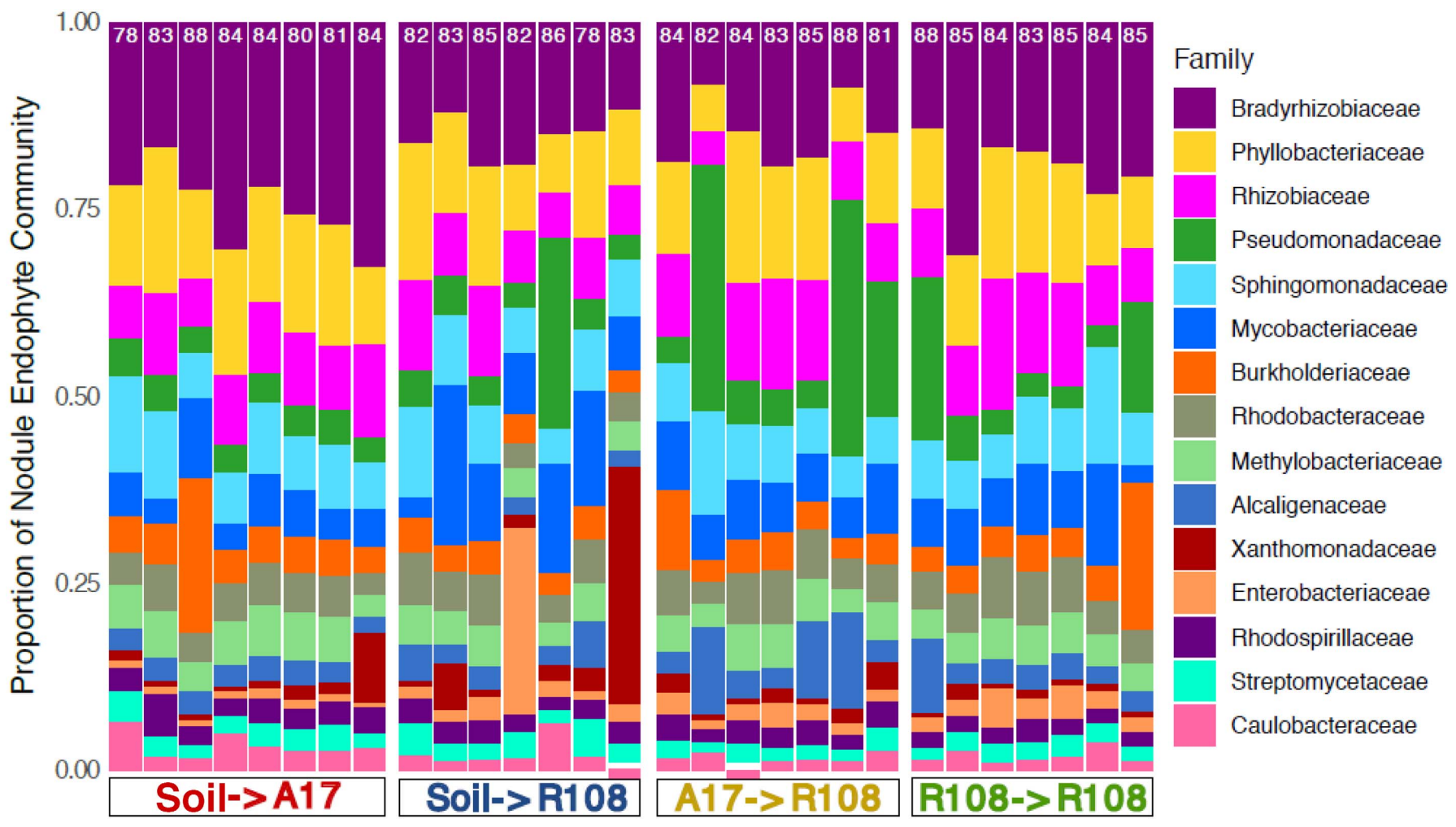

Fig. 3. Shifts in community composition of abundant bacterial families in nodules. Stacked bar plot of endophyte composition in nodules from each replicate pot. Families were considered abundant if they occurred with a mean frequency greater than 0.02 in at least one host genotype in the dataset generated from BLAST. Numbers above each bar denote the percentage of all endophytes present represented by these 15 families.
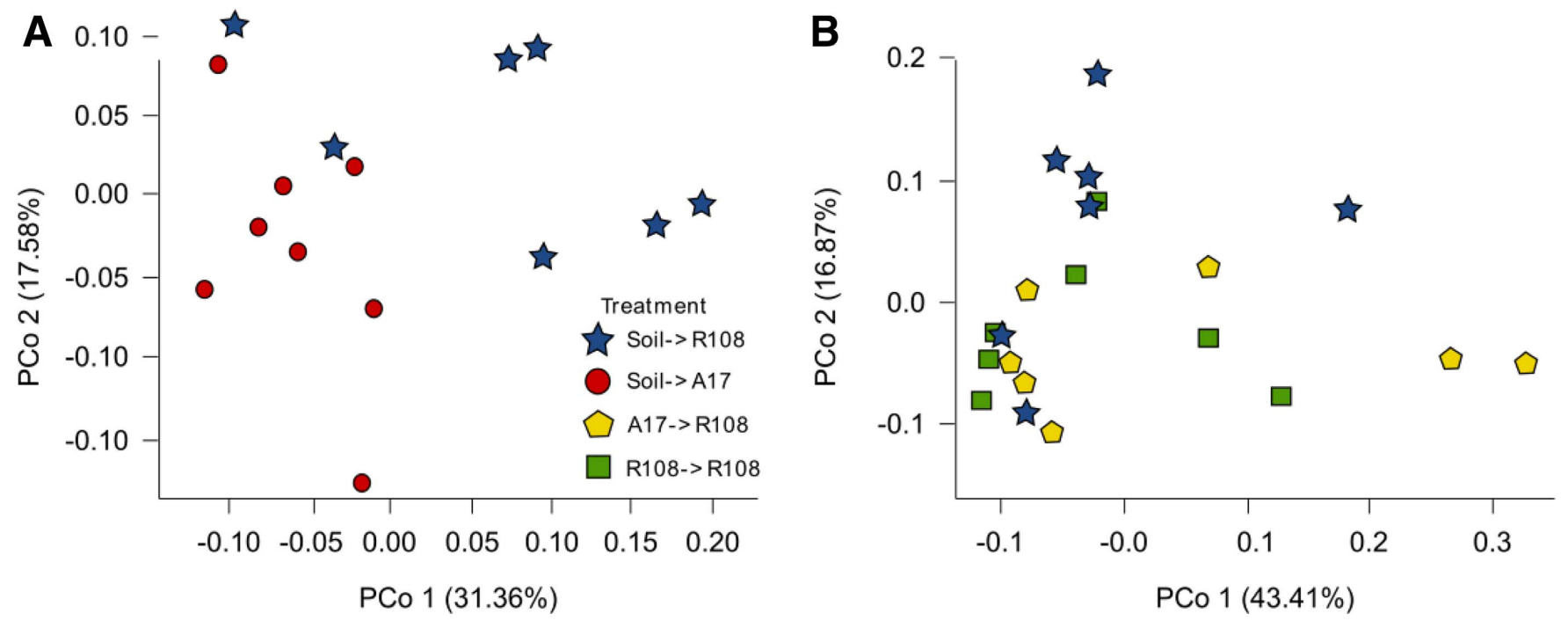

Fig. 4. Endophyte compositional comparison by principal coordinate (PCo) analysis of Bray-Curtis dissimilarity between A, host treatments and $\mathbf{B}$, legacy treatments. Treatments include soil $\rightarrow$ R108 (blue), soil $\rightarrow$ A17 (red), A17 $\rightarrow$ R108 (yellow), and R108 $\rightarrow$ R108 (green). The entire community dataset of $>400$ genera identified by BLAST was used for this figure. Outcomes of statistical tests can be found in Supplementary Table S1. 
Gutierrez et al. 2020), and Pseudomonas sp. 31-12 (Hynes et al. 2018) are known to be growth promoting, whereas $P$. aeruginosa is an opportunistic pathogen (Walker et al. 2004). Follow-up studies could investigate the prevalence of these species as well as whether their beneficial or detrimental properties in $M$. truncatula align with the same effects in other organisms.

Prior hosts can have significant legacy effects on Ensifer strain frequencies in root nodules (Burghardt et al. 2019), and on the entire microbial community of later generations (Kardol et al. 2007; Li et al. 2019; Meisner et al. 2013). However, this "legacy" selection does not extend to the nodule endophyte community composition more broadly. In this experiment, we found that neither the presence nor identity of a host in the previous generation significantly affected nodule endophyte community composition in a second generation. Although a small sample size, this result indicates that only the present host has a major effect on endophyte community composition in nodules. These compositional differences also extend to differences in the abundance of gene functional groups among plant genotypes. Interestingly, nearly half of the gene functional groups were shown to be expressed significantly differently between hosts, with most being enriched in the soil $\rightarrow$ R108 treatment (as compared with the soil $\rightarrow$ A17 treatment). Previous studies in the rhizosphere of other legumes (Mendes et al. 2014) and nonleguminous plants (Ofek-Lalzar et al. 2014) have shown the importance of membrane transport and nitrogen metabolism within the plant rhizosphere. The exclusion of rhizobia allowed us to focus on functions associated with nonrhizobial nodule endophytes, which could potentially reveal functions important to nonrhizobial endophytes within the rhizosphere and nodules. For instance, the nonoxidative branch of the pentose phosphate shunt is essential for the establishment of some mutualistic plant-microbe relationships (Hawkins et al. 2018), and degradation aromatic acid via the $\beta$-ketoadipate pathway underlies tomato pathogenicity of the
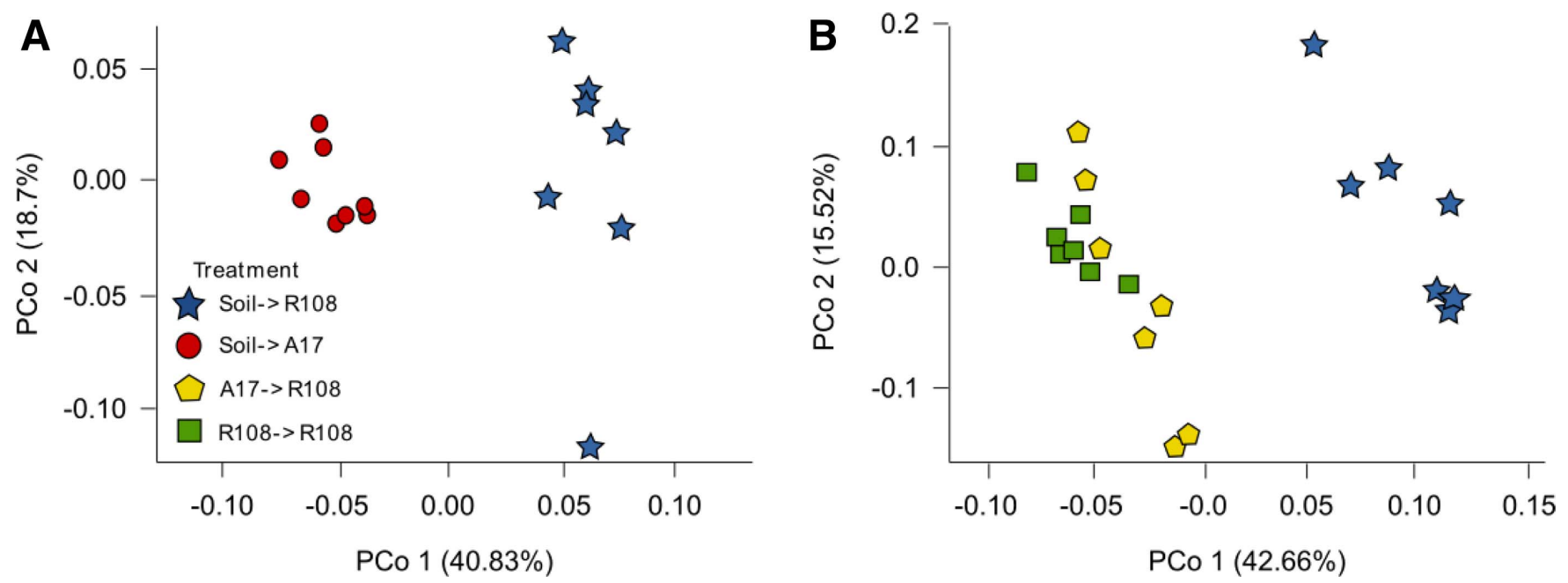

Fig. 5. Functional gene category abundance comparison by principal coordinate (PCo) analysis of Bray-Curtis dissimilarity between $\mathbf{A}$, host treatments and B, legacy treatments. Treatments include soil $\rightarrow R 108$ (blue), soil $\rightarrow A 17$ (red), A17 $\rightarrow R 108$ (yellow), and R108 $\rightarrow R 108$ (green). Functional gene categories were identified with MEGAN. Outcomes of statistical tests can be found in Supplementary Table S1.

TABLE 1

Functional gene category enrichment from MEGAN6 taxon identification pipeline ${ }^{a}$

\begin{tabular}{|c|c|c|c|c|c|}
\hline \multicolumn{3}{|c|}{ A17 versus $R 108$ enrichment } & \multicolumn{3}{|l|}{ Soil versus host legacy enrichment } \\
\hline$\beta$-Ketoadipate pathway & 0.000084 & 0.629 & ATP synthesis coupled proton transport & 0.000053 & 2.18 \\
\hline Carbohydrate catabolic process & 0.000162 & 1.8 & DNA repair & 0.000143 & 1.41 \\
\hline ATP synthesis coupled proton transport & 0.000646 & 1.88 & Sulfate transport & 0.000187 & 1.94 \\
\hline $\begin{array}{l}\text { 5-Phosphoribose 1-diphosphate biosynthetic } \\
\text { process }\end{array}$ & 0.00749 & 2.08 & Amino acid transmembrane transport & 0.000347 & 1.33 \\
\hline $\begin{array}{l}\text { Pentose-phosphate shunt, nonoxidative } \\
\text { branch }\end{array}$ & 0.0102 & 0.667 & Base-excision repair & 0.000413 & 1.5 \\
\hline ATP synthesis coupled electron transport & 0.012 & 4.17 & Branched chain amino acid biosynthetic process & 0.000436 & 3.67 \\
\hline
\end{tabular}

${ }^{a}$ The 10 most enriched functional gene groups between treatments. Soil $\rightarrow$ A17 versus soil $\rightarrow R 108$ is shown on the left and host $\rightarrow R 108$ versus soil $\rightarrow \mathrm{R} 108$ is shown on the right. All fold changes (FCs) display the enrichment in the soil $\rightarrow \mathrm{R} 108$ treatment. The $P$ values displayed have been adjusted using a Bonferroni correction. 
tomato wilt pathogen Fusarium oxysporum (Michielse et al. 2012). Thus, the enrichment of these functional groups may have consequences for host fitness.

Consistent with the findings of Burghardt et al. (2019) for rhizobial community composition, we found that the presence or absence of a host is a stronger driver of functional groups than the genotype of the previous host; it also suggests that functional group differences are not driven solely by differences in family-level composition. Our finding is interesting from an agricultural perspective because of the increasing use of legumes as winter and summer cover crops (Baraibar et al. 2020). Our results suggest that the addition of these legume cover crops to cropping rotation systems could influence the endophyte composition of the subsequent focal legume crops (e.g., soybean) (Bakker et al. 2018; Lang et al. 2019; Yuan et al. 2018).

Determining which plant host genes allow nodule inhabitation of beneficial mutualistic endophytes has wide-ranging implications across a variety of plant-related and agricultural fields such as increasing crop yield, growth, providing disease resistance, and many other benefits (Cordovez et al. 2019; Dini-Andreote 2020; Hubbard et al. 2019; Schlaeppi and Bulgarelli 2015). Some endeavors such as genome-wide association studies are already underway which can be used to identify how genetic variation among plant hosts maps to differences in the microbial community (Beilsmith et al. 2019). Analyzing the direct effects on host fitness of specific bacterial species inhabiting the root nodules would allow us to determine which species are plant growth promoting and should be selected for by genetically modified crops and other host plants. For example, coinoculation of Rhizobium spp. with various endophytes such as Bacillus spp. under certain conditions resulted in increased rhizobia proliferation as well as promoted plant growth in leguminous plants (Korir et al. 2017; Petersen et al. 1996). Searching for other similar synergies between endophyte species through coinoculation experiments could help create optimal inoculation communities for maximal crop production. Along with beneficial strains, legumes have been shown to form nodules with nonmutualistic rhizobia strains (Checcucci et al. 2016), and the same behavior may apply to the nodule endophyte community in general. Once host genes underlying nodule microbiome are identified, optimization via breeding programs or gene editing could be used to engineer plants to resist colonization by pathogens.

\section{ACKNOWLEDGMENTS}

We thank P. Tiffin for providing his lab resources and for consultation on our manuscript, as well as M. R. Wagner and R. Bledsoe for providing valuable input on early versions of our manuscript. Any opinions, findings, and conclusions or recommendations expressed in this material are those of the author(s) and do not necessarily reflect the views of the National Science Foundation.

\section{LITERATURE CITED}

Bakker, P. A. H. M., Pieterse, C. M. J., de Jonge, R., and Berendsen, R. L. 2018. The soil-borne legacy. Cell 172:1178-1180.

Bálint, M., Tiffin, P., Hallström, B., O’Hara, R. B., Olson, M. S., Fankhauser, J. D., Piepenbring, M., and Schmitt, I. 2013. Host genotype shapes the foliar fungal microbiome of balsam poplar (Populus balsamifera). PLoS One 8: e53987.

Baraibar, B., Murrell, E. G., Bradley, B. A., Barbercheck, M. E., Mortensen, D. A., Kaye, J. P., and White, C. M. 2020. Cover crop mixture expression is influenced by nitrogen availability and growing degree days. PLoS One 15: e0235868.

Beilsmith, K., Thoen, M. P. M., Brachi, B., Gloss, A. D., Khan, M. H., and Bergelson, J. 2019. Genome-wide association studies on the phyllosphere microbiome: Embracing complexity in host-microbe interactions. Plant J. 97:164-181.

Brown, S. P., Grillo, M. A., Podowski, J. C., and Heath, K. D. 2020. Soil origin and plant genotype structure distinct microbiome compartments in the model legume Medicago truncatula. Microbiome 8:139.

Bucciarelli, B., Hanan, J., Palmquist, D., and Vance, C. P. 2006. A standardized method for analysis of Medicago truncatula phenotypic development. Plant Physiol. 142:207-219.

Buchfink, B., Xie, C., and Huson, D. H. 2015. Fast and sensitive protein alignment using DIAMOND. Nat. Methods 12:59-60.

Burghardt, L. T., Epstein, B., Guhlin, J., Nelson, M. S., Taylor, M. R., Young, N. D., Sadowsky, M. J., and Tiffin, P. 2018. Select and resequence reveals relative fitness of bacteria in symbiotic and free-living environments. Proc. Natl. Acad. Sci. U.S.A. 115:2425-2430.

Burghardt, L. T., Epstein, B., and Tiffin, P. 2019. Legacy of prior host and soil selection on rhizobial fitness in planta. Evolution 73:2013-2023.

Checcucci, A., Azzarello, E., Bazzicalupo, M., Galardini, M., Lagomarsino, A., Mancuso, S., Marti, L., Marzano, M. C., Mocali, S., Squartini, A., Zanardo, M., and Mengoni, A. 2016. Mixed nodule infection in Sinorhizobium meliloti-Medicago sativa symbiosis suggest the presence of cheating behavior. Front. Plant Sci. 7:835.

Cloutier, M. L., Murrell, E., Barbercheck, M., Kaye, J., Finney, D., GarcíaGonzález, I., and Bruns, M. A. 2020. Fungal community shifts in soils with varied cover crop treatments and edaphic properties. Sci. Rep. 10:6198.

Cordovez, V., Dini-Andreote, F., Carrión, V. J., and Raaijmakers, J. M. 2019. Ecology and evolution of plant microbiomes. Annu. Rev. Microbiol. 73:69-88.

Costa-Gutierrez, S. B., Lami, M. J., María, C. C.-D. S., Zenoff, A. M., Vincent, P. A., Molina-Henares, M. A., Espinosa-Urgel, M., and de Cristóbal, R. E. 2020. Plant growth promotion by Pseudomonas Putida KT2440 under saline stress: Role of eptA. Appl. Microbiol. Biotechnol. 104: 4577-4592.

de Vasconcelos Martins Ferreira, L., de Carvalho, F., Fonseca Colombo Andrade, J., Padua Oliveira, D., Vasconcelos de Medeiros, F. H., and de Souza Moreira, F. M. 2020. Co-inoculation of selected nodule endophytic rhizobacterial strains with Rhizobium tropici promotes plant growth and controls damping off in common bean. Pedosphere 30:98-108.

Dini-Andreote, F. 2020. Endophytes: The second layer of plant defense. Trends Plant Sci. 25:319-322.

Egamberdieva, D., Wirth, S. J., Shurigin, V. V., Hashem, A., and Abd_Allah, E. F. 2017. Endophytic bacteria improve plant growth, symbiotic performance of chickpea (Cicer arietinum L.) and induce suppression of root rot caused by Fusarium solani under salt stress. Front. Microbiol. 8:1887.

Fischer, D. G., Chapman, S. K., Classen, A. T., Gehring, C. A., Grady, K. C., Schweitzer, J. A., and Whitham, T. G. 2014. Plant genetic effects on soils under climate change. Plant Soil 379:1-19.

Garcia, B. J., Labbé, J. L., Jones, P., Abraham, P. E., Hodge, I., Climer, S., Jawdy, S., Gunter, L., Tuskan, G. A., Yang, X., Tschaplinski, T. J., and Jacobson, D. A. 2018. Phytobiome and transcriptional adaptation of Populus deltoides to acute progressive drought and cyclic drought. Phytobiomes J. 2:249-260.

Haas, D., and Défago, G. 2005. Biological control of soil-borne pathogens by fluorescent pseudomonads. Nat. Rev. Microbiol. 3:307-319.

Hawkins, J. P., Ordonez, P. A., and Oresnik, I. J. 2018. Characterization of mutations that affect the nonoxidative pentose phosphate pathway in Sinorhizobium meliloti. J. Bacteriol. 200:e00436-17.

Heath, K. D., and Grillo, M. A. 2016. Rhizobia: Tractable models for bacterial evolutionary ecology. Environ. Microbiol. 18:4307-4311.

Heath, K. D., and Stinchcombe, J. 2013. Explaining mutualism variation: A new evolutionary paradox? Evolution 68:309-317.

Hol, W. H. G., Bezemer, T. M., and Biere, A. 2013. Getting the ecology into interactions between plants and the plant growth-promoting bacterium Pseudomonas fluorescens. Front. Plant Sci. 4:81.

Hubbard, C. J., Li, B., McMinn, R., Brock, M. T., Maignien, L., Ewers, B. E., Kliebenstein, D., and Weinig, C. 2019. The effect of rhizosphere microbes outweighs host plant genetics in reducing insect herbivory. Mol. Ecol. 28: 1801-1811.

Huson, D. H., Beier, S., Flade, I., Górska, A., El-Hadidi, M., Mitra, S., Ruscheweyh, H.-J., and Tappu, R. 2016. MEGAN Community EditionInteractive exploration and analysis of large-scale microbiome sequencing data. PLOS Comput. Biol. 12:e1004957.

Hynes, R. K., Dumonceaux, T. J., Kangsopa, J., and Town, J. R. 2018. Genome sequence of a plant growth-promoting Rhizobacterium, Pseudomonas sp. strain 31-12. Microbiol. Resour. Announce. 7:e00947-18. 
Ikeda, A. C., Bassani, L. L., Adamoski, D., Stringari, D., Cordeiro, V. K., Glienke, C., Steffens, M. B. R., Hungria, M., and Galli-Terasawa, L. V. 2013. Morphological and genetic characterization of endophytic bacteria isolated from roots of different maize genotypes. Microb. Ecol. 65:154-160.

Kardol, P., Cornips, N. J., van Kempen, M. M. L., Tanja Bakx-Schotman, J. M., and van der Putten, W. H. 2007. Microbe-mediated plant-soil feedback causes historical contingency effects in plant community assembly. Ecol. Monogr. 77:147-162.

Korir, H., Mungai, N. W., Thuita, M., Hamba, Y., and Masso, C. 2017. Co-inoculation effect of rhizobia and plant growth promoting rhizobacteria on common bean growth in a low phosphorus soil. Front. Plant Sci. 8:141.

Lang, M., Bei, S., Li, X., Kuyper, T. W., and Zhang, J. 2019. Rhizoplane bacteria and plant species co-determine Phosphorus-mediated microbial legacy effect. Front. Microbiol. 10:2856.

Leite, J., Fischer, D., Rouws, L. F. M., Fernandes-Júnior, P. I., Hofmann, A., Kublik, S., Schloter, M., Xavier, G. R., and Radl, V. 2016. Cowpea nodules harbor non-rhizobial bacterial communities that are shaped by soil type rather than plant genotype. Front. Plant Sci. 7:2064.

Li, X., Jousset, A., de Boer, W., Carrión, V. J., Zhang, T., Wang, X., and Kuramae, E. E. 2019. Legacy of land use history determines reprogramming of plant physiology by soil microbiome. ISME J. 13:738-751.

Long, S. R. 1989. Rhizobium-legume nodulation: Life together in the underground. Cell 56:203-214.

Martínez-Hidalgo, P., and Hirsch, A. M. 2017. The nodule microbiome: $\mathrm{N}_{2}$-fixing rhizobia do not live alone. Phytobiomes J. 1:70-82.

Meisner, A., De Deyn, G. B., de Boer, W., and van der Putten, W. H. 2013. Soil biotic legacy effects of extreme weather events influence plant invasiveness. Proc. Natl. Acad. Sci. U.S.A. 110:9835-9838.

Melnyk, R. A., Hossain, S. S., and Haney, C. H. 2019. Convergent gain and loss of genomic islands drive lifestyle changes in plant-associated Pseudomonas. ISME J. 13:1575-1588.

Mendes, L. W., Kuramae, E. E., Navarrete, A. A., van Veen, J. A., and Tsai, S. M. 2014. Taxonomical and functional microbial community selection in soybean rhizosphere. ISME J. 8:1577-1587.

Mhamdi, R., Mrabet, M., Laguerre, G., Tiwari, R., and Aouani, M. E. 2005. Colonization of Phaseolus vulgaris nodules by Agrobacterium-like strains. Can. J. Microbiol. 51:105-111.

Michielse, C. B., Reijnen, L., Olivain, C., Alabouvette, C., and Rep, M. 2012. Degradation of aromatic compounds through the $\beta$-ketoadipate pathway is required for pathogenicity of the tomato wilt pathogen Fusarium oxysporum f. sp. lycopersici. Mol. Plant Pathol. 13:1089-1100.
Morella, N. M., Weng, F. C.-H., Joubert, P. M., Metcalf, J. E., Lindow, S., and Koskella, B. 2020. Successive passaging of a plant-associated microbiome reveals robust habitat and host genotype-dependent selection. Proc. Natl. Acad. Sci. U.S.A. 117:1148-1159.

Mrabet, M., Mnasri, B., Romdhane, S. B., Laguerre, G., Aouani, M. E., and Mhamdi, R. 2006. Agrobacterium strains isolated from root nodules of common bean specifically reduce nodulation by Rhizobium gallicum. FEMS Microbiol. Ecol. 56:304-309.

Ofek-Lalzar, M., Sela, N., Goldman-Voronov, M., Green, S. J., Hadar, Y., and Minz, D. 2014. Niche and host-associated functional signatures of the root surface microbiome. Nat. Commun. 5:4950.

Petersen, D. J., Srinivasan, M., and Chanway, C. P. 1996. Bacillus polymyxa stimulates increased Rhizobium etli populations and nodulation when co-resident in the rhizosphere of Phaseolus vulgaris. FEMS Microbiol. Lett. 142:271-276.

Schlaeppi, K., and Bulgarelli, D. 2015. The plant microbiome at work. Mol. Plant-Microbe Interact. 28:212-217.

Sharaf, H., Rodrigues, R. R., Moon, J., Zhang, B., Mills, K., and Williams, M. A. 2019. Unprecedented bacterial community richness in soybean nodules vary with cultivar and water status. Microbiome 7:63.

Wagner, M. R., Lundberg, D. S., Del Rio, T. G., Tringe, S. G., Dangl, J. L., and Mitchell-Olds, T. 2016. Host genotype and age shape the leaf and root microbiomes of a wild perennial plant. Nat. Commun. 7:12151.

Walker, T. S., Bais, H. P., Déziel, E., Schweizer, H. P., Rahme, L. G., Fall, R., and Vivanco, J. M. 2004. Pseudomonas aeruginosa-plant root interactions. Pathogenicity, biofilm formation, and root exudation. Plant Physiol. 134:320-331.

Xiao, X., Chen, W., Zong, L., Yang, J., Jiao, S., Lin, Y., Wang, E., and Wei, G. 2017. Two cultivated legume plants reveal the enrichment process of the microbiome in the rhizocompartments. Mol. Ecol. 26:1641-1651.

Yuan, J., Zhao, J., Wen, T., Zhao, M., Li, R., Goossens, P., Huang, Q., Bai, Y., Vivanco, J. M., Kowalchuk, G. A., Berendsen, R. L., and Shen, Q. 2018. Root exudates drive the soil-borne legacy of aboveground pathogen infection. Microbiome 6:156.

Zgadzaj, R., Garrido-Oter, R., Jensen, D. B., Koprivova, A., Schulze-Lefert, P., and Radutoiu, S. 2016. Root nodule symbiosis in Lotus japonicus drives the establishment of distinctive rhizosphere, root, and nodule bacterial communities. Proc. Natl. Acad. Sci. U.S.A. 113:E7996-E8005.

Zhang, F., Berg, M., Dierking, K., Félix, M.-A., Shapira, M., Samuel, B. S., and Schulenburg, H. 2017. Caenorhabditis elegans as a model for microbiome research. Front. Microbiol. 8:485. 